\title{
Personal psychotherapy in psychiatric training: study of four London training schemes
}

\author{
AIMS AND METHOD \\ We carried out a web-based survey to \\ establish the proportion of London \\ psychiatry senior house officers who \\ undertake personal psychotherapy. \\ Demographics, training \\ characteristics and psychotherapy \\ experience were examined using \\ descriptive statistics. Predictors of \\ personal psychotherapy status \\ were examined using logistic \\ regression.
}

\author{
RESULTS \\ The majority of trainees who under- \\ took personal psychotherapy \\ included training as a reason for \\ doing so. Of the participants, $16 \%$ had \\ undergone personal psychotherapy \\ and of the remainder, $73 \%$ reported \\ that they would consider it in the \\ future. Ethnicity was the only pre- \\ dictor of psychotherapy status, with \\ White British trainees nearly four \\ times more likely to pursue it.
}

\begin{abstract}
CLINICAL IMPLICATIONS
Personal psychotherapy appeared to have relevance to training from both a pastoral and a learning perspective. If training schemes wish to support this practice they will need to address difficulties trainees may face in accessing therapy. Trainees might also be encouraged to think about the effect of cultural factors on their attitudes towards psychological therapies.
\end{abstract}

There has been a long-standing tradition of psychiatrists undergoing psychotherapy or psychoanalysis during training. However, figures from the USA suggests that although $70 \%$ of psychiatry trainees underwent psychotherapy in the 1950 s, ${ }^{1}$ only $32 \%$ of current trainees undertake personal psychotherapy. ${ }^{2}$ There are no data available for the UK and the uptake of personal psychotherapy here remains unknown. However, anecdotal evidence suggests a similar decrease in this country. Should this be the case, it may reflect a significant attitudinal shift regarding the utility of psychotherapy in the National Health Service (NHS) and the direction of psychiatric care.

Psychiatrists are increasingly asked to prescribe and undertake psychological therapies for a range of conditions ${ }^{3}$ and training in psychological therapies is thought to be essential. ${ }^{4}$ Some psychiatrists believe that personal psychotherapy has an important role in training, ${ }^{5,6}$ but there is much debate over the issue. According to one perspective, personal psychotherapy may provide the trainee with useful insights, experiential learning and support; other perspectives suggest it is lacking in evidence, costly and irrelevant. However, there is evidence that psychiatrists have high rates of stress ${ }^{7}$ compared with other doctors and that trainees are currently under particular strain. ${ }^{8}$ Doctors often find it hard to recognise and report their own illness, ${ }^{9}$ and some trainees may be using personal psychotherapy as a way of coping. There is also a view that psychotherapy may help clinicians differentiate their own psychopathology from that of their patients.

Some would also argue that since psychiatrists in training are expected to conduct psychodynamic psychotherapy with patients, ${ }^{10}$ they should conform to the same standards as those of trainee psychodynamic psychotherapists (i.e. mandatory personal psychotherapy). This would clearly have major financial and structural implications for training schemes.
Studies of the impact of personal psychotherapy on trainee therapists' practice have demonstrated no measurable effect on client outcomes, but a positive effect on therapists' empathy, warmth and genuineness. ${ }^{11}$ However, other studies have suggested that it may have a negative impact on client work because of an increase in trainees' emotional distress. ${ }^{11,12}$

A recent college debate on this subject revealed that many psychiatrists remain interested in this topic, and that many hold strong views. As such, this study was designed to explore this issue in more detail. The aims were to determine the popularity of personal psychotherapy among psychiatry senior house officers working in London, the types of therapy pursued, the number of senior house officers who would consider therapy, the reasons given for pursuing therapy, the guidance available and the factors that predicted the pursuit of therapy. In the current study the term 'personal psychotherapy' is used to refer to 'any psychological treatment ${ }^{\prime 13}$ where the psychiatrist was the client rather than the therapist.

\section{Method}

Ethical approval was obtained from the Barnet, Enfield \& Haringey Research Ethics Committee in London. Four of the eight psychiatry senior house officer training schemes in London were approached to participate in the study. The four schemes were chosen based on their geographical position, in order to gain as representative sample as possible. All senior house officers on these schemes at the time of the study were eligible to be participants except for those in stand-alone, non-training and general practitioner vocational training scheme posts. The survey was administered online and included a consent form, which was completed separately from the questionnaire to ensure anonymity. education \& training 
F

education \& training
As no appropriate, validated questionnaire was available, one was designed and created online by the authors using a web-based survey tool (www. surveymonkey.com). The first section contained questions on participants' sociodemographic details (age, gender, self-assigned ethnic category, religious affiliation and marital status), and their medical and psychiatric training. A second section explored their experience of personal psychotherapy. Those without experience of personal psychotherapy were asked whether they had previously considered it or would do so in future. The questionnaire was piloted with 20 junior doctors who had already completed senior house officer training. Following feedback and analysis, a number of minor changes were made to the questionnaire.

The participating training scheme administrators provided trainees' names and current training posts. They then sent an email to all trainees explaining the study and containing a link to the online questionnaire. Administrators sent a follow-up email 2-4 weeks later. Paper copies of the survey were sent to those without email addresses and to non-responders. Doctors' paper questionnaires that were returned to sender (indicating that the job list was incorrect) were removed from the final total number of questionnaires sent.

\section{Analysis}

The results were transferred from the survey website to the Statistical Package for the Social Sciences (SPSS) version 14.0 for Windows, double checked with printed copies of the responses and analysed. Demographics, training characteristics and psychotherapy experience were examined using descriptive statistics. Predictors of having or considering personal psychotherapy were examined using logistic regression.

\section{Results}

In total, 140 questionnaires were returned from a total of 294 senior house officers contacted. Seven questionnaires were returned to sender and deducted from the denominator leaving an overall response of $48.7 \%$.

\section{Demographic characteristics}

The mean age of participants was 29 years (s.d. $=3.2$ ) and the majority $(n=87,63 \%)$ of the sample were men. Just under half of respondents graduated from a medical school outside the UK, suggesting that ethnic minority groups had not been systematically excluded. The mean number of psychiatry training posts undertaken was 4.0 (s.d. = 2.0) (Table 1).

\section{Psychotherapy experience and views about therapy}

Twenty-two participants (16\%) had undertaken personal psychotherapy. The mean length of therapy was 15.2 months (range $0.2-104$, s.d. $=23.8$ ). Fifteen were currently in therapy.

The most common type of therapy was psychodynamic (45\%), with others including counselling, eclectic, group, systemic and cognitive-behavioural therapies. Frequency of therapy ranged between one session per week (68\% of participants) and four sessions per week. Of the trainees who had no direct experience of personal psychotherapy, 28 (26\%) stated that they had not considered psychotherapy and would never consider it.

Of the participants who undertook psychotherapy, $48 \%$ said it was for personal reasons, 39\% for both training and personal reasons, and $13 \%$ for training alone. Respondents who had undergone personal psychotherapy were asked if guidance in finding a therapist was available from training schemes. Only 5\% had received formal guidance. The remaining 95\% stated that they had received informal guidance.

Respondents were divided into two groups for subgroup comparison purposes (Group A and B). Group A comprised those with direct experience of therapy or

Table 1. Demographic and training characteristics of participants $(n=140)$

Characteristic n $(\%)$

Men $^{a}$ 87 (63)

Marital status ${ }^{b}$

Single $60 \quad(43)$

Married $56(40)$

Long-term relationship

Separated/divorced

Ethnic group ${ }^{b}$

White ${ }^{c}$

$63(45)$

Black/Black British

10 (7)

Asian/Asian British

51 (37)

Chinese

2 (1)

Mixed race

Other

$7 \quad(5)$

$6 \quad(4)$

Religious/spiritual beliefs ${ }^{d}$

Religion during upbringing $\quad 77 \quad$ (55)

Religious beliefs currently $48 \quad$ (34)

Spiritual beliefs currently 13 (9)

Neither spiritual nor religious $\quad 35 \quad$ (25)

Country of graduatione

UK

Training scheme A

Training scheme B

Training scheme $C$

Training scheme D

MRCPsych status ${ }^{b}$

No parts yet passed 62 (44)

Part I passed

Part II passed

a. Data for two participants missing

b. Data for one participant missing.

c. Includes White British, White Irish and White Other.

d. Data for 45 participants missing.

e. Training schemes indicate those graduating outside the UK. Data for two participants missing. 
those stating that they had considered it in the past or would consider it in the future, whereas Group B comprised those that had no experience of personal psychotherapy and said they would never consider it (Table 2).

Sociodemographic and training characteristics were combined into binary variables for subgroup analysis. Ethnicity was the only significant predictor of attitudes to personal psychotherapy. White British respondents, the largest single ethnic group, were found to be four times more likely than others to be in Group A (Table 3).

\section{Discussion}

Psychiatry trainees are the consultants of the future, and many will go on to prescribe psychotherapy and manage psychotherapy services. As such, their experience of, and attitudes towards, psychotherapy will determine the place of psychotherapy in the NHS.

We were primarily interested in whether the practice of personal psychotherapy really mattered to trainees, training schemes or to psychiatry in general. The fact that only $16 \%$ of participants had direct experience of their own psychotherapy may suggest that it is merely of interest to a minority. However, our findings also indicated that many trainees had or would consider psychotherapy for themselves, suggesting that many felt it had some value or role.

Table 2. Psychotherapy experience

\begin{tabular}{|lc}
\hline & $n(\%)$ \\
\hline Currently in therapy & $15(11)$ \\
Therapy ever & $22(16)$ \\
No therapy ever but have considered in past ${ }^{a}$ & $44(41)$ \\
No therapy ever but would consider in future & $79(67)$ \\
Group A & $111(79)$ \\
Group B & $28(20)$ \\
Missing values & $1(1)$ \\
\hline
\end{tabular}

a. Percentages are of respondents who have not had direct experience of therapy.

b. Respondents who have ever had therapy, are having therapy, plus those who have no experience of therapy but have considered it in the past or would do so in future.

c. Respondents who have never had and are not having therapy, and would not consider it in the future.
So, given that many participants felt personal psychotherapy has a value, what was the nature of the value added? We initially hypothesised that personal psychotherapy was providing support or an acceptable form of treatment for these doctors. However, the study raised the possibility that trainees have other reasons for undertaking therapy. More than $50 \%$ of the participants in the study who were in psychotherapy gave training as one of the reasons for undertaking therapy, and some cited training as the only reasons for doing so (13\%). This raises the question of what personal psychotherapy may be providing, which is not being provided elsewhere in training. In the psychoanalytic world, personal psychotherapy is a compulsory part of training, and is thought to provide important experiential learning. This experiential learning may be the value trainees are interested in, especially in a profession where reflective practice is encouraged. Furthermore, in our new competency-based trainings, there may be few other opportunities to experience this degree of reflective learning.

Having established that personal psychotherapy has a pastoral and training value, we were also interested in the popularity of personal psychotherapy and what this suggest for the future. In the absence of any historical data from the UK, we can only speculate as to whether our finding of $16 \%$ uptake is a decline. However, comparison with the USA prevalence (32\%) does suggest that the practice of personal psychotherapy is less popular in the UK than in the USA.

Given that the majority of our participants said they would consider psychotherapy, we were curious as to why so few had pursued it. In order to answer this question, we explored the predictors of personal psychotherapy among participants. The study found that the only significant predictor was ethnicity. This finding may have reflected valid cultural differences, but may also have indicated inequalities in access to personal psychotherapy. If this is the case, training schemes may wish to think about how they can address this matter, to counter the impact this may have on our trainees and their attitudes to psychotherapy.

\section{Limitations}

With a modest response rate, the findings of this survey must be interpreted with some caution. In particular, the finding of $16 \%$ prevalence for uptake of personal

Table 3. Predictors of respondents being in Group A, derived from logistic regression analysis ${ }^{\mathrm{a}}$

\begin{tabular}{|c|c|c|c|}
\hline Age (below/above mean) & 0.802 & 1.123 & $0.453-2.783$ \\
\hline Ethnic group (White British/all others) & 0.009 & 3.761 & $1.386-10.204$ \\
\hline MRCPsych status (passed no parts/passed one or both parts) & 0.438 & 0.697 & $0.280-1.736$ \\
\hline Constant & 0.076 & 0.048 & \\
\hline
\end{tabular}

a. Religion had to be removed from the equation owing to too many missing values.

Significant result in bold. 
F

education \& training psychotherapy may be an overestimate of the true uptake due to the influence of self-selection bias.

Another limitation of the study is that the sample was unlikely to be representative of all trainees in England. However, since the participants came from all geographical regions of London, the results are likely to be representative of those in London. We can also postulate that personal psychotherapy may be more common among London trainees than those located elsewhere, due to the greater availability of psychotherapy and the proximity to psychotherapy training institutions. It will be important to repeat the study in other regions to establish the psychotherapy experience and attitudes nationwide. The range of experience reported suggests that the sample mirrored the population under investigation. There was a good spread of age and gender in the study population and the fact that half of the sample graduated outside the UK and half described themselves as White British, indicates a culturally diverse sample.

\section{Use of an online survey tool}

One strength of the study was the use of the online survey for data collection. This is a relatively novel technique in the NHS and proved accessible, relatively inexpensive and efficient. In the near future, online methods may be a useful way of reaching larger populations and improving response rates.

\section{Acknowledgements}

This work is dedicated to the memory of Dr Richard Lucas OBE who supported and inspired us. We would also like to thank DrTina Rothi and the Research Support Department at the University of Hertfordshire for their helpful input and advice.

\section{Declaration of interest}

None.

\section{References}

1 Holt RR, Luborsky L. Personality patterns of psychiatrists. Menninger Foundation 1958; 2 20-7.

2 Moran, M. Is personal psychotherapy still crucial for residents? Psychiatr News 2007; 42: $6-26$

3 National Institute for Health and Clinical Excellence. Depression Clinical Guidance CG23. NICE, 2004 (amended in 2007).

4 Agarwal S, SinghY, Palanisamy V Basker R, Van der Speck R. Psychotherapy requirements as recommended by the College: awareness and achievement by senior house officers. Psychiatr Bull 2007: 31: 394-6.

5 Mace $C$. Personal therapy in psychiatric training. Psychiatr Bull 2001; 25: 3-4.

6 Hollins S. 'Experience in psychotherapy is a necessary part of psychiatric training.' Debate,

Royal College of Psychiatrists Annual General Meeting, Edinburgh, 2005.

7 Fothergill A, Edwards D, Burnard P. Stress, burnout, coping and stress

management in psychiatrists: findings from a systematic review. Int J Soc Psychiatry 2004; 50 $54-65$.

8 Lydall GJ, Malik A, Bhugra D. Mental health of applicants seems to be deteriorating. BM J 2007; 334: 1335.

9 McKevitt C, Morgan M. Illness doesn't belong to us. JR Soc Med 1997; 90: 491-5.

10 Royal College of Psychiatrists. Requirements for Psychotherapy Training as Part of Basic Specialists PsychiatricTraining. Royal College of Psychiatrists, 2002 (http://www. rcpsych.ac.uk/PDF/ptBasic.pdf)

11 Macran S, Shapiro DA. The role of personal therapy for therapists: a eview. BrJ Med Psychol 1998; 71: $13-25$.

12 Dubovsky SL, ScullyJH. Hazards of long-term psychotherapy during psychiatric residency. Psychiatry 1990; 53: 185-94.

13 Gelder M, Gath D, Mayou R, Cowen P. Oxford Textbook of Psychiatry (3rd edn). Oxford University Press, 1996.

*Deborah Dover Specialist Registrar in Child \& Adolescent Psychiatry, Child and Family Department, Tavistock Centre, 120 Belsize Lane, London NW3 5BA, email: deborah.dover@gmail.com,Ed Beveridge Consultant Psychiatrist, St Charles Hospital, London, Gerry Leavey Research and Development Department, St Ann's Hospital, London, Michael King Department of Mental Health Sciences, University College London, UK 